\title{
Recent Advances and Surgical Applications of Monoclonal Antibodies
}

\author{
Selim Elnemr, Ahmed Abd El Aal Sultan, Samh Massoud Saad Nassar
}

\author{
Department of General Surgery, Faculty of Medicine, Al-Azhar University, Cairo, Egypt
}

*Corresponding author: Samh Massoud Saad Nassar, Mobile: (+20) 1019136662, E-Mail: samehnassar7@gmail

\begin{abstract}
Background: Monoclonal antibodies plays an important role in surgical disease practice specially cancers, Crohn's Disease and ulcerative colitits. Monoclonal antibodies is a type of biological therapy that utilizes a person's natural immune defense system to fight diseases. Monoclonal antibodies for cancers is essentially the stimulation of immune system via a variety of reagents such as vaccines infusion of $\mathrm{T}$ cells or cytokines. These reagents reacted through one of several mechanisms: 1- By stimulating the antitumor response either by increasing the number of effector cells or be production one or more soluble mediators such as lymphokines. 2- By decreasing suppressors mechanisms. 3- By altering tumor cells to increase their immunogenicity and make them more susceptible to immunological defenses. 4- By improving tolerance to cytotoxic drugs or radiotherapy such as stimulating bone marrow function with granulocyte colony stimulating factor (G- CSF). Monoclonal antibodies is a type of immunotherapy but there is another types of immunotherapy such as nonspecific immunotherapies and cancer vaccines mechanism of monoclonal antibodies in surgical disease.
\end{abstract}

Objective: This work was aimed to study the role of monoclonal antibodies in surgical disease as a new tool to surgical armamentarium.

Conclusion: It could be concluded from this study that monoclonal antibodies helps the body to fight disease and infection. Monoclonal antibodies are used to treat many types of carcinoma and cancers.

Keywords: Surgical Applications, Monoclonal Antibodies

\section{INTRODUCTION}

Monoclonal

antibodies are immunoglobulin molecules (IgG, IgM, IgA, IgE) that are secreted from a population of identical cells. The commercial utility of these highly specific molecules lies in the simplicity by which large quantities of IgG or other antibodies with identical binding sites are generated by in vivo or in vitro methods ${ }^{(\mathbf{1})}$.

Over the last decade, anti-epidermal growth factor receptor (EGFR) monoclonal antibodies (mAbs) have been firmly established as essential drugs for the treatment of metastatic colorectal cancer (CRC). Cetuximab and panitumumab have been approved by American and European drug agencies ${ }^{(2)}$.

Monoclonal antibodies are an indispensable cornerstone of clinical oncology. Notably, all FDA-approved antibodies comprise the IgG class, although numerous research articles proposed monoclonal antibodies of the IgM, IgG, IgA and IgE classes directed specifically against tumor-associated antigens. In particular, for the IgE isotype class, several recent studies could demonstrate high tumoricidic efficacy ${ }^{(3)}$.
Since their development more than 30 years ago, monoclonal antibodies have taken their place as established and effective cancer therapies. Early investigations into the effectiveness of monoclonal antibodies were disappointing: the murine origins of the first monoclonal antibodies led to host-immune reactions against them, leading to rapid clearance of the antibodies and rendering treatment possibly ineffective, and in some cases, dangerous. Since then, advances in technology, especially in the development of chimeric and humanized antibodies, have greatly improved their therapeutic applications (4).

Based on the work of Köhler and Milstein ${ }^{(1)}$, who produced the first monoclonal antibody (mAb) in 1975, numerous mAbs have been approved for treatment of various diseases by the US Food and Drug Administration (FDA) in oncology (alemtuzumab, rituximab, ofatumumab, bevacizumab), rheumatology (tocilizumab, adalimumab, golimumab), gastroenterology (infliximab, certolizumab pegol), dermatology (efalizumab, ustekinumab) 
and transplant rejection prevention (daclizumab, basiliximab) ${ }^{(\mathbf{5})}$.

Challenges still exist, however results to date have demonstrated weak activity with these agents as monotherapies, suggesting that combinations of targeted agents and other therapies will be necessary to achieve improved outcomes. Heterogeneous antigen expression, restricted penetration of the targeting agent into the tumor, limited tumor toxicity, or even resistance to the targeting agent might be some of the problems encountered in a monotherapy setting. Targeted radionuclide therapy (TRT) may be a promising way to improve targeted treatment of head and neck cancer ${ }^{(6)}$.

\section{Aim of the Work}

This work was aimed to study the role of monoclonal antibodies in surgical disease as a new tool to surgical armmentrium.

\section{Monoclonal Antibodies}

Antibodies are proteins produced by an individual in response to the presence of a foreign molecule in the body. These foreign molecules are known as antigens, and they usually result from invading organisms such as bacteria, fungi or viruses. Antibodies bind to antigens and elicit a range of effector mechanisms to destroy the invading organism. Monoclonal antibodies are immunoglobulin molecules (IgG, IgM, IgA, IgE) that are secreted from a population of identical cells (i.e., cloned cells). The commercial utility of these highly specific molecules lies in the simplicity by which large quantities of IgG or other antibodies with identical binding sites are generated by in vivo or in vitro methods ${ }^{(7)}$.

In 1975 Kohler and Milstein described a method for the 'production of antibodies of predefined specificity'. This technical breakthrough allowed, for the first time, the production of antibody molecules of a single specificity which could be characterized and defined. Such monoclonal antibodies immediately became valuable research tools, and applications in the diagnosis and therapy of human disease began to be widely investigated. A second technical revolution has now arrived in the ability to manipulate antibody genes and to design and produce antibody molecules tailor-made for their application. Such redesigned antibody molecules are now rapidly becoming valuable reagents for therapy of human diseases as well as improved diagnostics and research reagents ${ }^{(7)}$.

\section{Monoclonal Antibodies in Research and Diagnostic Applications Introduction:}

Antibodies have proved to be invaluable reagents for the detection and quantitation of many types of substances both in vitro and in vivo and have, therefore, found wide application in both the research laboratory and the diagnostics industry. Many of the techniques used in these two situations are similar, and thus it is helpful to review them together. In a chapter of this length it is impossible to cover all the ways that MAbs have been used in these areas; thus the emphasis in this chapter is on applications which are particularly widely used and those which are likely to benefit most from the ability to design engineered or modified antibodies ${ }^{(8)}$.

\section{Immunoassays in diagnostics and research:}

Immunoassays are carried out to detect and quantitate the presence of a particular antigen or antibody in a test fluid. The development of immunoassays began with polyclonal antisera and thus precedes the introduction of MAbs. However, today MAbs are widely used, and many assays routinely used in, for example, hospital clinical biochemistry laboratories which rely on them. In fact, MAbs have found widespread use in laboratories of all types, in applications from drug discovery to detecting drugs of abuse in athletes and horses. In contrast to polyclonal antisera, production of MAbs allows a potentially unlimited supply of identical reagent which can be selected from a number of different clones to have the optimal characteristics for the intended assay. For some assay formats such as radioimmunoassay, however, polyclonal antisera remain the best reagents, as they are frequently of higher apparent affinity than MAbs ${ }^{(4)}$.

Therefore, it is useful to examine the types of immunoassays to appreciate the role of monoclonal antibody reagents. Some of the properties of MAbs of interest for immunoassay development are given in Table $1^{(9)}$.

Table 1: Properties of monoclonal antibodies important for immunoassay design Unlimited quantity of identical antibody ${ }^{(9)}$ 
Antibodies to distinct epitopes can be selected from panels of clones

Easily purified to reduce background binding

Readily digested to produce fragments with equivalent specificity

Can be produced to impure antigen

Often of lower apparent affinity than polyclonal antisera

\section{Surgical Application of Monoclonal Antibodies \\ Breast cancer: \\ Adjuvant treatment of breast cancer: impact of monoclonal antibody therapy directed against the HER2 receptors.}

The use of chemotherapy and endocrine therapies as adjuncts to the treatment of earlystage breast cancer has yielded small but significant improvements in disease-free and overall survival. Increased understanding of the role of growth factor receptors enabled the rational development of agents that are capable of modulating their function. A humanized monoclonal antibody to the HER2 receptor, trastuzumab, has demonstrable single-agent activity in metastatic breast cancer and enhances the anti tumour effects of chemotherapy. As a consequence, trastuzumab has been tested in the adjuvant setting the results of which have been presented recently. This review briefly summaries the use of trastuzumab in advanced breast cancer and describes recent studies of its use in the adjuvant setting. The development of trastuzumab and its application in the treatment of breast cancer is now often quoted as the paradigm for translational research. In other words, increased understanding of tumor biology is yielding more effective, less toxic, targeted therapies. The large benefits attributable to trastuzumab have not previously been seen with any other agent in the past. The challenge for the future is how to incorporate it into clinical practice for the benefit of patients (10).

Effects on quality of life of combined trastuzumab and chemotherapy in women with metastatic breast cancer.

After completion of chemotherapy, patients treated with trastuzumab and chemotherapy reported significant improvement in fatigue $(\mathrm{P}+\mathrm{ADw}-. \mathrm{05})$ as compared with their baseline scores. Higher proportions of patients receiving the combined therapy achieved improvement in global QOL (P +ADw-. 05) than did patients treated with chemotherapy alone. Higher proportions of the combined therapy group also achieved improvement in physical and role functioning and in fatigue as compared with the chemotherapy group, but the differences were not statistically signi? cant. There were no differences in the proportions of patients in the two groups that reported worsening. Conclusion: Statistically signi? cantly higher proportions of patients treated with a combination of trastuzumab and chemotherapy reported improved global QOL than did patients treated by chemotherapy alone ${ }^{(\mathbf{1 1})}$.

\section{Role of subcutaneous formulation of trastuzumab in the treatment of patients with positive breast cancer.}

Agents targeting human epidermal growth factor receptor 2 (HER2) represent the cornerstone of systemic treatment of patients with HER2-positive breast cancer, irrespective of the stage at presentation. Trastuzumab, monoclonal antibody against HER2, is the most commonly used anti-HER2 agent. Trastuzumab is often administered as a single agent for prolonged periods of time, and the need for repeated dosing represents a significant logistic problem. Subcutaneous formulation of trastuzumab represents a response to the challenge posed by the need for repeated dosing of the drugs. It was demonstrated in a randomized clinical trial that subcutaneous administration of trastuzumab is noninferior to intravenous delivery in terms of efficacy as well as pharmacokinetics. Moreover, prospective data from another randomized trial indicate that subcutaneous injection is preferred by the patients over intravenous administration ${ }^{(12)}$.

\section{Ulcerative colitis:}

Tumor necrosis factor alpha blocking agents for induction of remission in ulcerative colitis (Review):

Tumor necrosis factor alpha blocking agents for treatment of active ulcerative colitis. Ulcerative colitis is a chronic relapsing inflammatory disorder of the large bowel. Although corticosteroids are effective for treating ulcerative colitis, approximately $20 \%$ of patients who respond become sick again when steroids are withdrawn and become steroid dependent. Furthermore, corticosteroids 
exhibit significant adverse effects. Tumor necrosis factor alpha (TNF) is a inflammatory cytokine that is involved in the pathogenesis of rheumatoid arthritis, Crohn's disease and psoriasis. TNF blocking drugs may provide an alternative treatment for patients who do not respond to corticosteroid and/or immunosuppressive drug treatment. This review shows that intravenous infusions of infliximab, a TNF blocking agent is effective in inducing clinical remission, promoting mucosal healing, and reducing the need for colectomy in patients with active ulcerative colitis whose disease has not responded to conventional treatment. In patients with moderate to severe ulcerative colitis whose disease is refractory to conventional treatment using corticosteroids and/or immunosuppressive agents, infliximab is effective in inducing clinical remission, inducing clinical response, promoting mucosal healing, and reducing the need for colectomy at least in the short term. Serious adverse events attributable to infliximab were not common in the included studies but physicians should be aware of and be prepared to deal with potential adverse events such as anaphylactic reactions and infections ${ }^{(\mathbf{1 3})}$.

\section{Pancreatic cancer: \\ Emerging antibodies for the treatment of pancreatic cancer:}

Pancreatic ductal adenocarcinoma cancer (PDAC) is the fourth leading cause of cancer death worldwide. Recently, two chemotherapy regimens have proven to improve median overall survival in comparison with gemcitabine. Based on better understanding of tumor molecular biology and of the role of tumor microenvironment, monoclonal antibodies (mAbs) could be an interesting and new type of targeted treatment of PDAC. After a decade of failures of targeted agents in advanced pancreatic cancer seems to see some novelties. The advancement in our knowledge of the complex biology of the tumors with the identification of relevant pathways such as NOTCH and Wnt as well as driver mutations led to the development of new therapeutic agents. Obviously, we need to expect the results from phase III trials. Therefore, the advancement in safety, identification of adequate sequence and combination of treatment options, and predictive biomarkers should be the principal research goals for the future. It is not the light at the end of the tunnel but probably it could be the real first step ahead and a new history in the treatment of this lethal malignancy ${ }^{(\mathbf{1 4})}$.

\section{Prostate cancer:}

- Advances in the therapy of prostate cancer induced bone disease

Prostate cancer calls are characterized by an exquisite tropism for the bone, which translates into one of the highest rates of bone metastasis and skeletal morbidity. New effective treatments have emerged from a better understanding of the pathophysiology of bone metastasis. Prevention and treatment of bone metastases and ADT-induced bone loss is essential for the management of men with aggressive PCa. Bone metastasis and SREs are the first metastatic site and source of morbidity, respectively RANKI signalling is a key regulator for osteoclast-mediated bone destruction in both normal bone remodeling and pathologic conditions. In addition, RANKL contributes to the vicious cycle of bone destruction and tumor growth in PCa. Inhibition of RANKL using RANKL antibodies or RANK fusion proteins resulted in a prolonged inhibition of bone resorption and diminished $\mathrm{PCa}$ progression in bone in postmenopausal women and a preclinical animal model, respectively. Recently, a multicenter randomized open label active controlled phase 2 study demonstrated that denosumab a fully human monoclonal antibody against RANKL reduced the incidence of SREs. In a study done to summarize current insights and future perspectives in the therapy of prostate cancer induced bone disease. Showed that denosumab reduced skeletal related events in patients with bone metastasis from prostate cancer. In addition, the potential rule of denosumab in the management of treatment induced bone loss and the prevention of bone metastasis is currently under investigations ${ }^{(\mathbf{1 5})}$.

\section{Colorectal cancer:}

- Epidermal growth factor receptor (EGFR) inhibitors for metastatic colorectal cancer (Review)

Bowel (colorectal) cancer is the third most common cancer worldwide. The International Agency for Research on Cancer estimated a crude colorectal cancer incidence rate of 1,361,000 in 2012 (with 694,000 deaths), with $55 \%$ of cases occurring in highincome countries ${ }^{(16)}$. Although improvements 
in treatment, particularly over the last 10 years, have brought significant improvements in survival, metastatic colorectal cancer (mCRC) remains a major cause of morbidity and mortality. Epidermal growth factor receptor (EGFR) inhibitors prevent cell growth and have shown benefit in the treatment of metastatic colorectal cancer, whether used as single agents or in combination with chemotherapy. Clear benefit has been shown in trials of EGFR monoclonal antibodies (EGFR mAb) but not EGFR tyrosine kinase inhibitors (EGFR TKI). However, there is ongoing debate as to which patient populations gain maximum benefit from EGFR inhibition and where they should be used in the metastatic colorectal cancer treatment paradigm to maximize efficacy and minimize toxicity ${ }^{(17)}$.

Comparing EGFR MAb to bevacizumab in combination with standard therapy in KRAS exon 2 wildtype (WT) populations, progression-free survival and overall survival are not improved, but tumour response rate is increased. The odds of overall grade 3 to 4 toxicity are increased with EGFR MAb compared with bevacizumab. In practice, this does not change the treatment paradigm in a country where both EGFR MAb and bevacizumab are available without restriction.In the RASWT population, one is usually used in the ?rst line in combination with chemotherapy, and the other is used in combination with another chemotherapy on progression (e.g. FOLFIRI with cetuximab first line, then FOLFOX with bevacizumab second line,or FOLFIRI with bevacizumab first line, then FOLFOX with cetuximab second line). The choice of line in which EGFR MAb is used, and the chemotherapy partner it is used with, remain up to clinician preference at this point given the lack of definitive evidence showing that choice of oxaliplatin or irinotecan affects EGFR MAb efficacy ${ }^{(17)}$.

Considering the other areas of investigation, there is no evidence that either EGFR $\mathrm{mAb}$ vacizumab is superior in combination with chemotherapy. A full discussion of sequencing of the seagents for optimal benefit is again outside the bounds of this review. Nevertheless, assuming that the agents are of equal cost, there is no evidence to support restriction of EGFR MAb to a particular line of therapy or bind its provision to prior bevacizumab exposure (or lack thereof).
However, it is clear that the addition of EGFRI to the combination of chemotherapy and bevacizumab in people with KRAS exon 2 WT metastatic colorectal cancer does not improve progression-free survival, overall survival, or tumour response rate but does increase rates of toxicity (overall grade 3 to 4 toxicity, grade 3 to 4 diarrhoea and rash) and may cause harm. The use of EGFR MAb in addition to the combination of chemotherapy and bevacizumab is therefore not supported by the current data. Similarly, there is current lyno evidence to support the use of EGFR tyrosine kinase inhibitors in metastatic colorectal cancer (whether in KRAS WT or MT populations), and their use should remain investigational at present ${ }^{(\mathbf{1 7})}$.

\section{DISCUSSION}

Monoclonal antibodies are immunoglobulin molecules (IgG, IgM, IgA, $\operatorname{IgE})$ that are secreted from a population of identical cells. The commercial utility of these highly specific molecules lies in the simplicity by which large quantities of IgG or other antibodies with identical binding sites are generated by in vivo or in vitro methods ${ }^{(\mathbf{1})}$.

The generation of human mAbs is often desirable for clinical applications. This is because murine antibodies are recognised as foreign when administered to humans and therefore elicit an immune response directed against the administered MAb: a human antimouse antibody response or HAMA. This prevents repeat administration of MAb as the HAMA results in formation of immune complexes which are rapidly cleared, rendering the antibody ineffective. In addition, HAMA responses may result in adverse reactions by the patient such as allergic reactions, hepatic dysfunction and in some cases anaphylactic shock. Attempts to overcome this problem have driven much research both in reconstructing rodent antibodies by genetic engineering and in attempts to generate human MAbs. Human antibodies may also be advantageous in that they are compatible with human effector mechanisms such as complement and ADCC which are often not fully activated by rodent antibodies ${ }^{(\mathbf{1 8})}$.

As with tyrosine kinase inhibitors such as gefitinib and erlotinib, cetuximab and panitumumab commonly cause a moderate to severe, dose-dependent so-called "acneiform" or papulopustular eruption in $50-100 \%$ of 
patients (19). Cardiac adverse events have occurred with at least 4 of the mAbs approved for cancer therapy. Cardiopulmonary arrest and/or sudden death resulted in $2 \%$ (4) of 208 patients given cetuximab and exposed to radiation therapy. No reactions were seen in 212 patients given radiation alone. Cardiac arrhythmias have been reported for rituximab, cardiomyopathy following trastuzumab, and left ventricular dysfunction (LVD) for pertuzumab, trastuzumab, and trastuzumab emtansine ${ }^{(20)}$.

Tumor lysis syndromeoccur $48-72 \mathrm{~h}$ after the start of anticancer chemotherapy as a result of the rapid, drug-mediated death of large numbers of malignant cells. This results in hyperkalemia, hypercalcemia, hyperphosphatemia, and hyperuricemia, a profound ionic imbalance and a possible progression to acute renal failure, cardiac arrhythmias, seizures, and death ${ }^{(\mathbf{2 1})}$.

Progressive

multifocal

leukoencephalopathy is a progressive, usually fatal viral disease that in some respects resembles multiple sclerosis, as the myelin sheath of nerve cells is ultimately destroyed affecting transmission of nerve impulses ${ }^{(22)}$.

In vivo diagnostics: The use of antibodies for imaging disease has been developed to the point where there are now several products marketed for clinical use (Table 10) and many more under development. Antibodies to suitable antigens can be used to confirm the presence and extent of disease in the body, identify its location and monitor the progress of any therapy. To achieve this the antibody is labelled with a suitable radioisotope, usually a short half-life gamma emitter, and the emissions from the isotope detected with a gamma camera ${ }^{(\mathbf{1 3})}$.

Radioimmunodetection of human tumours: A major impetus to the development of RAID has been the desire to develop techniques to allow detection of human tumours and determine the extent and location of disease, particularly monitoring metastatic spread. In many cases accurate, early diagnosis using RAID can be a contributor to effective treatment, with the major use of RAID being to monitor disease in patients following treatment by surgery, radiotherapy or chemotherapy ${ }^{(13)}$.

Tumour-associated antigens: Useful tumour-associated antigens are absent on normal tissues and present at high levels on tumour cells, preferably homogeneously on all cells of the tumour ${ }^{(23)}$.

Radioimmunoguided surgery (RIGS) may prove to be a useful aid to surgery for the removal of tumour tissue. Patients are preadministered radiolabelled antibody, and a hand-held gamma detecting probe is used intraoperatively to locate occult tumour which may otherwise escape detection. Some studies have suggested that the sensitivity obtained with RIGS may be better than with radioimmunoscintigraphy ${ }^{(23)}$, and RIGS has been shown to improve assessment of tumour spread and improve surgical intervention. For example, in patients with primary colorectal tumours RIGS with the anti-TAG72 antibody CC49 allowed detection of $86 \%$ of tumours, whereas in patients undergoing secondlook surgery for recurrent disease $97 \%$ of tumour deposits were detected ${ }^{(24)}$, the sensitivity of detection apparently being linked to antigen expression. Most studies have used antibody labelled with the weak gamma emitter 125I, although $99 \mathrm{mTc}$-labelled antibody has also been successfully tested ${ }^{(25)}$.

Non-tumour radioimmunodetection: MAb-based radioimmuno detection has also been applied in several other disease situations, where precise imaging may offer useful clinical information. These include detection of myocardial necrosis, imaging of blood clots and the detection of infection and inflammation.

Breast cancer: A humanized monoclonal antibody to the HER2 receptor, trastuzumab, has demonstrable single-agent activity in metastatic breast cancer and enhances the anti tumour effects of chemotherapy. As a consequence, trastuzumab has been tested in the adjuvant setting the results of which have been presented recently. the use of trastuzumab in advanced breast cancer exceed expectations of investigators, clinicians and patients alikeThe development of trastuzumab and its application in the treatment of breast cancer is now often quoted as the paradigm for translational research. In other words, increased understanding of tumour biology is yielding more effective, less toxic, targeted therapies. The large benefits attributable to trastuzumab have not previously been seen with any other agent in the past. The challenge for the future is how to incorporate it into clinical practice for the benefit of patients (10). 
Ulcerative colitis: chronic relapsing inflammatory disorder of the large bowel. Tumour necrosis factor (TNF) blocking drugs may provide an alternative treatment for patients who do not respond to corticosteroid and/or immunosuppressive drug treatment. That intravenous infusions of infiximab, a TNF blocking agent is effective in inducing clinical remission, promoting mucosal healing, and reducing the need for colectomy in patients with active ulcerative colitis whose disease has not responded to conventional treatment. TNF blocking agents are effective for induction of remission in refractory Crohn's disease and recent reports have suggested that they may have a similar role in the management of ulcerative colitis controlled trials which have addressed this question. The main reported outcomes were clinical response, clinical remission and endoscopic remission measured by validated disease activity indices, as well as adverse events.Anti-TNF agents have been shown to be effective for the induction of remission in Crohn's disease ${ }^{(\mathbf{1 3})}$.

Pancreatic cancer: Pancreatic ductal adenocarcinoma cancer (PDAC) is the fourth leading cause of cancer death worldwide. Recently, monoclonal antibodies (mAbs) could be an interesting and new type of targeted treatment of PDAC. After a decade of failures of targeted agents in advanced pancreatic cancer seems to see some novelties. The advancement in our knowledge of the complex biology of the tumors with the identification of relevant pathways led to the development of new therapeutic agents. Therefore, the advancement in safety, identification of adequate sequence and combination of treatment options, It is not the light at the end of the tunnel but probably it could be the real first step ahead and a new history in the treatment of this malignancy ${ }^{(\mathbf{1 4})}$.

Prostate cancer: Calls are characterized by an exquisite tropism for the bone, which translates into one of the highest rates of bone metastasis and skeletal morbidity. New effective treatments have emerged from a better understanding of the pathophysiology of bone metastasis. Prevention and treatment of bone metastases essential for the management denosumab a fully human monoclonal antibody against prostate cancer and bone metastasisdenosumab reduced skeletal related events in patients with bone metastasis from prostate cancer. In addition, the potential rule of denosumab in the management of treatment induced bone loss and the prevention of bone metastasis is currently under investigations ${ }^{(\mathbf{1 5})}$.

Colorectal cancer: Bowel (colorectal) cancer is the third most common cancer worldwide. The International Agency for Research on Cancer estimated a crude colorectal cancer incidence rate of $1,361,000$ in 2012 (with 694,000 deaths), with 55\% of cases occurring in high-income countries (16). Although improvements in treatment, particularly over the last 10 years, have brought signi? cant improvements in survival, metastatic colorectal cancer (mCRC) remains a major cause of morbidity and mortality. Epidermal growth factor receptor (EGFR) inhibitors prevent cell growth and have shown benefit in the treatment of metastatic colorectal cancer, whether used as single agents or in combination with chemotherapyvacizumab is superior in combination with chemotherapy and bevacizumab is therefore ${ }^{(\mathbf{1 7})}$.

\section{Conclusion}

It could be concluded from this study that monoclonal antibodies helps the body to fight disease and infection

Monoclonal antibodies are used to treat many types of carcinoma and cancers.

That are minimize risk of spreading such as Crohn's Disease and ulcerative colitis monoclonal antibodies also can lessen the side effects from other cancers treatments such as chemotherapy and also concluded that monoclonal antibodies may be given after surgery either alone or as an adjuvant therapy in combination with chemotherapy and radiotherapy monoclona antibodies are usually given be intravenous. It may also be used to minimize the side effects often caused by chemotherapy and radiation therapy. monoclonal antibodies are also used by itself to treat cancers but they are most often used along with or after another type of treatment to post its effects lastly we can concluded that the future of monoclonal antibodies are used armenterum of surgical interventions.

Surgical applications of monoclonal antibodies available and has been the subject of many studies which support its effects however, the cost of these monoclonal antibodies is high. And there is a risk of systemic reaction as a result of this limitation scientists have tried to modify the monoclonal antibodies uses in surgical applications to lower this allergiencity 
and increase their immunogenicity. In this way lower doses of less reactive allergens could induce tolerance with much lower incidence of side effects and perhaps greater convenience finally, monoclonal antibodies have good roles in recent clinical research, diagnosis and treatment of surgical disease.

\section{REFERENCES}

1. Köhler G and Milstein C (1975): Continuous cultures of fused cells secreting antibody of predefined specificity. Nature, 256:495-497.

2. You B and Chen EX (2012): AntiEGFR monoclonal antibodies for treatment of colorectal cancers: development of cetuximab and panitumumab. J Clin Pharmacol., 52(2):128-155.

3. Jensen-Jarolim $\mathbf{E}$ and Pawelec $\mathbf{G}$ (2012): The nascent field of Allergo Oncology. Cancer Immunol Immunother., 61: 1355-1357.

4. Daniels PB, Fletcher JE, O'neill PM, Stafford CG, Bacarese-Hamilton $T$ and Robinson GA (1995): A comparison of three fluorophores for use in an optical biosensor for the measurement of prostate-specific antigen in whole blood. Sensors Actuators, B26: 447-451.

5. Rommer PS, Dudesek A, Stüve $O$ and Zettl UK (2014): Monoclonal antibodies in treatment of multiple sclerosis. Clin Exp Immunol., 175(3):373-384.

6. Nestor M (2009): Targeted radionuclide therapy in head and neck cancer. Head Neck, 32: 666-678.

7. King DJ, Antoniw P, Owens RJ, Adair JR, Haines AM, Farnsworth AP, Finney $H$ and Lawson AD (1995): Preparation and preclinical evaluation of humanised A33 immunoconjugates for radioimmunotherapy. Br J Cancer, 72: 1364-1372.

8. Kricka LJ (1993): Ultrasensitive immunoassay techniques. Clin Biochem., 26: 325-331.

9. Larson SM (1995): Improving the balance between treatment and diagnosis: a role for radioimmunodetection. Cancer Res., 55: 5756-5758.

10. Simonds HM and Miles D (2007). Adjuvant treatment of breast cancer: impact of monoclonal antibody therapy directed against the HER2 receptor. Expert Opinion on Biological Therapy, 7(4): 487-491.

11. Osoba D, Slamon DJ, Burchmore M and Murphy $M$ (2002): Effects on quality of life of combined trastuzumab and chemotherapy in women with metastatic breast cancer. J Clin Oncol., 20(14):3106-3113.

12. Weinblatt ME, Schiff $M$, Valente $R$, van der Heijde D, Citera G, Zhao C, Maldonado $M$ and Fleischmann $R$ (2013): Head-to-head comparison of subcutaneous abatacept versus adalimumab for rheumatoid arthritis. Arthritis Rheum., 65: 28-38.

13. Lawson MM, Thomas AG and Akobeng AK (2006): Tumour necrosis factor alpha blocking agents for induction of remission in ulcerative colitis. Cochrane Database Syst Rev., 3:CD005112.

14. Sohal DP, Mangu PB, Khorana AA, Shah MA, Philip PA, O'Reilly EM, Uronis HE, Ramanathan RK, Crane CH, Engebretson A, Ruggiero JT, Copur MS, Lau M, Urba S and Laheru D (2016): Metastatic Pancreatic Cancer: American Society of Clinical Oncology Clinical Practice Guideline. J Clin Oncol., 34(23):2784-2796.

15. Heidenreich A, Bastian PJ, Bellmunt J, Bolla M, Joniau S, van der Kwast T, Mason M and Matveev V (2014): EAU guidelines on prostate cancer. part 1: screening, diagnosis, and local treatment with curative intent-update 2013. Eur Urol., 65(1):124-137.

16. Ferlay J, Soerjomataram I, Dikshit R, Eser S, Mathers C, Rebelo $M$ and Parkin DM (2015): Cancer incidence and mortality worldwide: sources, methods and major patterns in GLOBOCAN 2012. Int J Cancer, 136(5):359-386.

17. Chan DLH, SegelovE, Wong RSH, Smith A, Herbertson RA, Li BT, TebbuttN, Price $T$ and Pavlakis $N$ (2017): Epidermal growthfactor receptor (EGFR) inhibitors for metastaticcolorectal cancer. Cochrane Database Syst Rev., 6:CD007047. 
18. Adair JR and Bright SM (1995): Progress with humanised antibodies - an update. Exp Opin Invest Drugs, 4: 863-870.

19. Segaert $S$ and Van Cutsem $E$ (2005): Clinical signs, pathophysiology and management of skin toxicity during therapy with epidermal growth factor receptor inhibitors. Ann Oncol., 16:142533.

20. Baldo BA and Pham NH (2013): Adverse reactions to targeted and nontargeted chemotherapeutic drugs with emphasis on hypersensitivity responses and the invasive metastatic switch. Cancer Metastasis Rev., 32(3-4):723761.

21. Howard SC, Jones DP and Pui CH (2011): The tumor lysis syndrome. $\mathrm{N}$ Engl J Med., 364:1844-1854.

22. Brooks B (2007): Progressive multifocal leukoencephalopathy. In: Gilman S (ed). Neurobiology of Disease. London: Elsevier Academic Press; Pp. 185-196.
23. Hinkle GH, Mojzisik CM, Loesch JA, Hill TL, Thurston M.O., Sampsel J, Olsen J and Martin EW (1991): The evolution of the radioimmunoguided surgery system: an innovative technique for the intraoperative detection of tumour. Antibody Immunoconj Radiopharm., 4: 339-358.

24. Arnold MW, Schneebaum S, Berens A, Petty L, Mojzisik C, Hinkle G and Martin EW (1992): Intraoperative detection of colorectal cancer with radioimmunoguided surgery and CC49, a second generation monoclonal antibody. Ann Surgery, 216: 11-16.

25. Ind TE, Granowska M, Britton KE, Morris G, Lowe DG, Hudson CN and Shepherd JH (1994): Preoperative radioimmunodetection of ovarian carcinoma using a hand-held gamma detection probe. Br J Cancer, 70: 12631266.

26. 